\title{
Grand-Canonical simulation of 4D simplicial quantum gravity 目
}

\author{
S.Horata $^{a}$, H.S.Egawa ${ }^{b}$ and T.Yukawac \\ ${ }^{a}$ Department of Particle and Nuclear Physics, School of Mathematical Science, The Graduate \\ University for Advanced Studies, Tsukuba, Ibaraki 305-0801, Japan \\ ${ }^{\mathrm{b}}$ Department of Physics, Tokai University, Hiratsuka, Kanagawa 259-1292, Japan \\ ${ }^{\mathrm{c}}$ Coordination Center for Research and Education, The Graduate University for Advanced Studies, \\ Hayama, Miura, Kanagawa 240-0193, Japan
}

A thorough numerical examination for the field theory of 4D quantum gravity (QG) with a special emphasis on the conformal mode dependence has been studied. More clearly than before, we obtain the string susceptibility exponent of the partition function by using the Grand-Canonical Monte-Carlo method. Taking thorough care of the update method, the simulation is made for $4 \mathrm{D}$ Euclidean simplicial manifold coupled to $N_{X}$ scalar fields and $N_{A} \mathrm{U}(1)$ gauge fields. The numerical results suggest that $4 \mathrm{D}$ simplicial quantum gravity (SQG) can be reached to the continuum theory of $4 \mathrm{D}$ QG. We discuss the significant property of $4 \mathrm{D}$ SQG.

\section{Introduction}

Until now 4D SQG has been investigated from several points of view, such as the phase structure, nature of the transition, effects of additional matter fields, and the modified measure action 11. Unfortunately the numerical results could not resolve the problem for the existence of the continuum theory of 4D QG clearly. Gradually the original enthusiasm to explore 4D QG has been toned down. However, we remind that many problems about 4D SQG are yet to be studied.

As one of those problems, we pay attention to additional matter effects in $4 \mathrm{D}$ Euclidean SQG (Eucl.SQG). According to the recent numerical simulations, the string susceptibility exponent $\left(\gamma^{(4)}\right)$ takes a negative value with adding a few $\mathrm{U}(1)$ gauge fields. It makes drastic change of the phase diagram, and continuous phase transition with one gauge matter has been found [2]. Moreover, the numerically obtained $\gamma^{(4)}$ as a function of the number of matter fields turned out to be approximately equal to that of the prediction of $4 \mathrm{D}$ conformal field theory [3].

In order to see more definitely how the matter fields act on 4D Eucl.SQG, we repeat the study with employing the Grand-Canonical method

\footnotetext{
* presented by S.Horata
}

used for 2D SQG[四, which has given the satisfactory agreement of two results. For discussing the continuum theory of $4 \mathrm{D}$ QG based on the numerical analysis, we pay attention to the functional form of the partition function, which we expect enough to gain information for selecting the correct theory of gravity, if it exists at all. For obtaining the functional form of the partition function, we need to perform a simulation with varying the space volume. In practice, we calculate the probability $\left(P\left(N_{4}\right)\right)$ as a function of the volume $\left(N_{4}\right)$ by the Grand-Canonical method of the dynamical triangulation (DT) and estimate $\gamma^{(4)}$ from the scaling behavior of $P\left(N_{4}\right)$.

\section{Model and Grand-Canonical method}

Let us explain our model and the numerical method of the Grand-Canonical simulation. We consider a system of the simplicial gravity coupled to $\mathrm{U}(1)$ gauge fields and massless scalar fields. The action $(S)$ is given on $4 \mathrm{D}$ simplicial manifold as

$S=S_{G}+S_{A}+S_{X}$,

where $S_{G}, S_{A}$ and $S_{X}$ denote the action for the gravity, $\mathrm{U}(1)$ gauge fields $(A)$ and scalar fields $(X)$, respectively. 
For the gravity part, we use the discretized Einstein-Hilbert action in $4 \mathrm{D}$,

$S_{G}\left[\kappa_{2}, \kappa_{4}\right]=\kappa_{4} N_{4}-\kappa_{2} N_{2}$,

where $N_{i}$ denotes the number of $i$-simplex. The two parameters, $\kappa_{2}$ and $\kappa_{4}$, correspond to the inverse of the gravitational constant and the cosmological constant, respectively. The action for the $N_{X}$ scalar fields $X_{i}^{a}, a=1 \ldots N_{X}$, on the vertex $i$ is given by

$S_{X}=\sum_{a=1}^{N_{X}} \sum_{i j} o\left(l_{i j}\right)\left(X_{i}^{a}-X_{j}^{a}\right)^{2}$,

where $o\left(l_{i j}\right)$ is the number of four-simplices sharing a link $l_{i j}$. And the action for the $N_{A}$ vector field $A_{i j}^{a}\left(a=1 \ldots N_{A}\right)$ on link $l_{i j}$ reads

$S_{A}=\sum_{a=1}^{N_{A}} \sum_{i j k} o\left(t_{i j k}\right)\left(A^{a}\left(l_{i j}\right)+A^{a}\left(l_{j k}\right)+A^{a}\left(l_{k i}\right)\right)^{2}$,

where $o\left(t_{i j k}\right)$ is the number of four-simplices sharing a triangle $t_{i j k}$. These actions give the correct thermo-dynamical limit for large $N_{4}$ because they are proportional to $N_{4}$.

In order to simplify the calculation, the MonteCarlo simulations have been performed with the Canonical method previously. This method requires that $N_{4}$ fluctuates around the given target volume $\left(V_{4}\right)$. Thus, the volume fixing term $(\delta S)$ is added to the total action Eq.(11),

$\delta S\left(N_{4}, V_{4}\right)=\delta \kappa_{4}\left(N_{4}-V_{4}\right)^{2}$,

where $\delta \kappa_{4}$ is the free parameter. However, the Canonical method has limitations about the finite size effect and the ergodic property. Furthermore, the functional form of the size dependence of the partition function cannot be given clearly. For the improvement of numerical accuracy we perform the simulations varying the space volume by the Grand-Canonical method. The Grand-Canonical partition function with the matter fields is given as

$$
\begin{aligned}
& Z\left(\kappa_{2}, \kappa_{4}, N_{A}, N_{X}\right)=\sum_{T} e^{-S_{G}\left(\kappa_{2}, \kappa_{4}\right)} \\
& \prod_{N_{A}, N_{X}}\left(\int \prod_{l \in T} d A_{l} e^{-S_{A}\left(A_{l}\right)} \int \prod_{i \in T} d X_{i} e^{-S_{X}\left(X_{i}\right)}\right),
\end{aligned}
$$

where all geometries $T$ and matter fields $A, X$ are updated using the Metropolis method keeping the topology of discretized surface to be $S^{4}$.

From the analogy of the 2D case, we assume that the partition function obeys the KPZ like formula for the large- $N_{4}$ limit as

$Z \approx \sum_{N_{4}} N_{4}^{\gamma^{(4)}\left(\kappa_{2}\right)-3} e^{\left(\kappa_{4}-\kappa_{4}^{c}\left(\kappa_{2}\right)\right) N_{4}}$.

Then the probability $P\left(N_{4}\right)$ for the large- $N_{4}$ behaves approximately as

$P\left(N_{4}\right) \propto N_{4}^{\gamma^{(4)}-3} e^{-\left(\kappa_{4}-\kappa_{4}^{c}\right) N_{4}}$.

By tuning $\kappa_{4}$ close to $\kappa_{4}^{c}$, the exponential growth is suppressed and $P\left(N_{4}\right)$ is given as the power function of $N_{4}$. The parameter $\kappa_{4}$ is determined in the following manner: If $\kappa_{4}$ is chosen to be less (or greater) than $\kappa_{4}^{c}$, the system will expand (or shrink) exponentially in the Grand-Canonical simulation. By requiring an exponential increase (or decrease) to disappear in the simulation, we can find $\kappa_{4}$ precisely with the following form,

$\frac{\ln P\left(N_{4}\right)}{\ln N_{4}}=\left(\gamma^{(4)}-3\right)-\left(\kappa_{4}-\kappa_{4}^{c}\right) \frac{N_{4}}{\ln N_{4}}+O\left(1 / \ln N_{4}\right) .(9)$

After tuning $\kappa_{4}$, we generate the configurations constraining $N_{4}$ within an upper- and a lowerbound $\left(N_{4}^{\min } \leq N_{4} \leq N_{4}^{\max }\right)$.

\section{Numerical result}

In this section, we report numerical results with the Grand-Canonical method. In order to obtain $P\left(N_{4}\right)$, we allow $N_{4}$ to fluctuate between $N_{4}^{\text {min }}=1000$ and $N_{4}^{\max }=10000$. In Fig.1. we show $P\left(N_{4}\right)$ versus $N_{4}$ at $\kappa_{4}$ close to $\kappa_{4}^{c}$. The numerical result shows that $P\left(N_{4}\right)$ is given as a power function of $N_{4}$. It is suggested that the partition function in $4 \mathrm{D}$ obeys the KPZ type partition function as the $2 \mathrm{D}$ case.

We can also compute $\gamma^{(4)}$ from the slope of $P\left(N_{4}\right)$. Then, we show the parameter $(b)$, which is calculated from $\gamma^{4}$,

(6) $\gamma^{(4)}=-\frac{b}{2}\left(1-\frac{4}{b}+\sqrt{1-\frac{4}{b}}\right)$. 


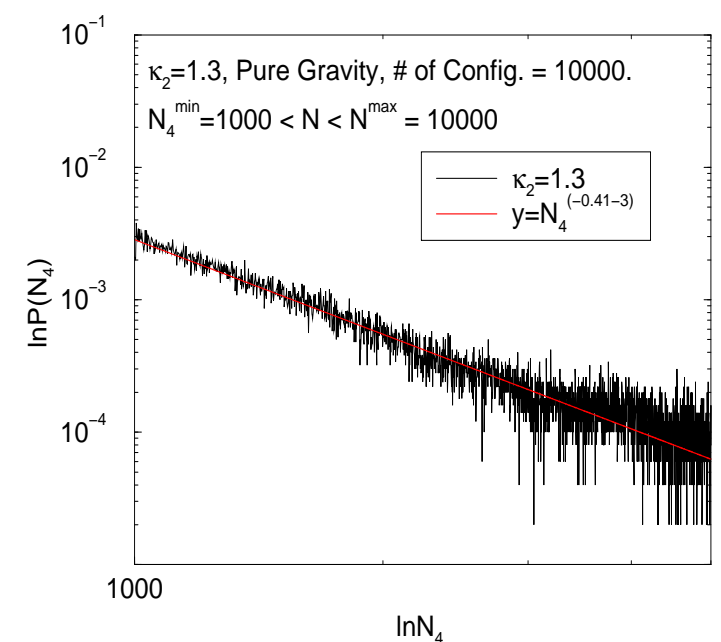

Figure 1. We plot $P\left(N_{4}\right)$ versus $N_{4}$ with log-log scale.

This relation is given from the analogy of $2 \mathrm{D}$ QG and the parameter $b$ is similar to the central charge in 2D QG. In Fig.2, we plot $b$ versus the number of matter fields, $N_{X}+62 N_{A}$, on the critical point $\left(\kappa_{2}^{c}\right)$ between the crumpled phase and the smooth phase. We compare $b$ obtained by two different numerical methods, i.e. the Canonical method with the MINBU algorithm and the Grand-Canonical method. Error bars include both the errors in determining $\kappa_{2}^{c}$ and the statistical errors, and we found that the former method has much larger errors than the latter method. We made the parameter fit of the data of the Grand-Canonical method by a linear function, $b=0.0030(3)\left(N_{X}+62 N_{A}\right)+3.98(3)$. The slope $b$ is approximately equal to the analytical result [5, 6.

\section{Summary and Discussion}

Let us summarize and discuss the main points made in the previous section. From the numerical results of the Grand-Canonical method, we find the KPZ type partition function also appears in 4D QG. The string susceptibility exponent $\gamma^{(4)}$ obtained from the size dependence of the partition function can be obtained by the GrandCanonical method rather accurately. The matter contribution to $b$, which is computed from $\gamma^{(4)}$ with the analogy of $2 \mathrm{D} \mathrm{QG}$, turns out to be ap-

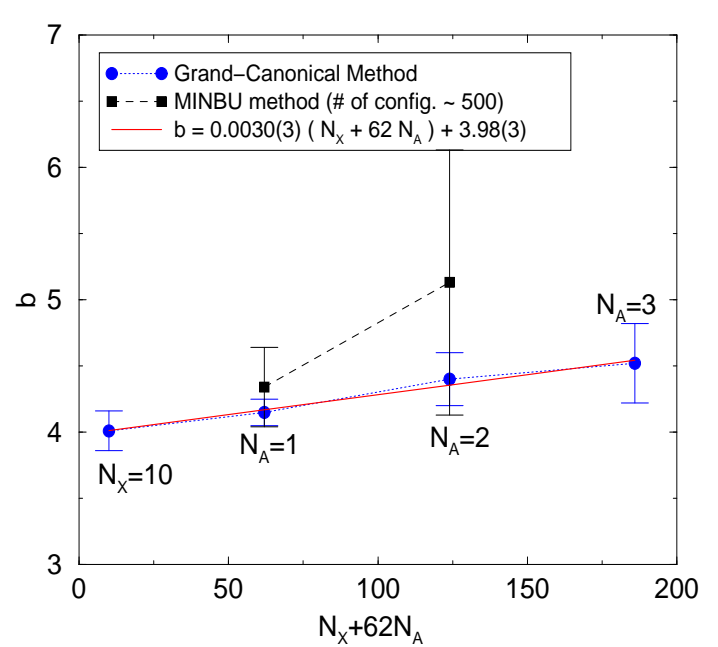

Figure 2. We plot $b$ versus the number of the matter fields, $N_{X}+62 N_{A}$.

proximately equal to the conformal part [5, [6]. Our numerical results suggest that it may be possible to understand the continuum theory of $4 \mathrm{D}$ QG from the analogy of $2 \mathrm{D}$ QG. In order to look more details on the relation to the continuum theory of $4 \mathrm{D}$ QG, these results encourage us to continue investigation by the Grand-Canonical method and push more efforts on the analytical calculation of the conformal gravity at the same time.

\section{REFERENCES}

[1] G. Thorleifsson, Nucl. Phys. B (Proc. Suppl.) 73 (1998) 133.

[2] H. S. Egawa, S. Horata, N. Tsuda and T. Yukawa, Prog. Theor. Phys. 106 (2001) 1037.

[3] H. S. Egawa, S. Horata, and T. Yukawa, Nucl. Phys. B (Proc. Suppl.) 106-107 (2002), 971.

[4] S. Oda, N. Tsuda and T. Yukawa, Nucl. Phys. B (Proc. Suppl.) 63 (1998) 733

[5] H. S. Egawa, S. Horata, and T. Yukawa, Prog. Theor. Phys. submitted.

[6] K. Hamada, Prog. Theor. Phys. 103 (2000) 1237, Prog. Theor. Phys. 105 (2001) 673. 